\title{
Rupture Dynamics and Seismological Variables in Earthquake
}

\author{
Jeevan Regmi \\ Department of Physics Prithvi Narayan Campus, Pokhara
}

Email: jsregmi28@gmail.com

\begin{abstract}
The Earthquake results by the movement of tectonic plates. There might be various causes of motion of these plates. Based on type of motion and speed of collision, the nature and strength of earthquake varies. It is difficult to present an unique model of physics to explain the earthquake since the nature and strength significantly varies with time and place.

Keywords: Tectonic plates, fault, Epicenter, Seismic wave, Average slip, Rupture dynamics, Fault lubrication, Elastohydrodynamics, Seismological variables.
\end{abstract}

\section{INTRODUCTION}

An earthquake is a motion of the ground surface ranging from a faint tremor to a wild motion capable of shaking everything. The earthquake is a form of energy of wave motion transmitted through the surface layer of the earth in widening circles from a point of sudden energy release- the focus. The waves so produced are seismic waves that travel outward in all directions from the focus gradually losing the energy. These waves are the result of sudden movement of faults beneath the earth surface. The rocks on both sides of the fault slowly bents for a large period of time due to continuous application of tectonic forces on the movement of large crystal masses. This process causes to accumulate the energy in the bent rocks and when the critical point is reached, the strain is relieved by slippage on the fault and a large amount of energy is instantaneously released in the form of seismic waves. As the plates slide past each other, relative motion is sometimes accommodated by a relatively constant gradual slip, at the rate of few millimeters per year while at other times; the accumulated strain is released in earthquakes with a slip rate of the order of meters per second.

\subsection{Theoretical Explanation}

Earthquake physicists attempt to link the available observations to the processes occurring in Earth's deep interior to help them interpret as long term and short term processes.

\subsection{Long-term processes}

There are two major forces in Earth's crust, the shear stress and the shear strain. The shear stress is the force per unit area applied tangent to a plane whereas the shear strain is a dimensionless quantity that describes the distortion of a body in response to a shear stress.
Recent progress in space-based geodesy, made possible by the global positioning system and satellite interferometry, now provide us with a clear pattern of crustal movement and strain accumulation. The relative plate motion determined from various data shows to be about $2-7 \mathrm{~cm} / \mathrm{year}$, which translates into a strain accumulation rate of approximately $3 \times 10^{-7} / \mathrm{y}$ along plate boundaries. The strain also accumulates in plate interiors, but at a much slower rate of about $3 \times 10^{-8} / \mathrm{y}$ or less. Since the rigidity of the crustal rocks is about $3 \times 10^{4} \mathrm{MPa}$, this corresponds to a stress accumulation rate of $10^{-2} \mathrm{MPa} / \mathrm{y}$ along plate boundaries, an order of magnitude less in plate interiors.

When the stress at a point in the crust exceeds a critical value, called the local strength, a sudden failure occurs. The plane along which failure occurs is called the fault plane and the point where failure initiates is called the focus. Typically, there is a sudden displacement of the crust at the fault plane following the failure, and elastic waves are radiated. This is an earthquake. For most earthquakes, the displacement occurs at an existing geological fault, that is, a plane that is already weak.

\subsection{Short-term processes and friction}

Earthquake fault motion can be viewed as frictional sliding on a fault plane. The friction changes due to relative displacement of the two sides of the fault plane, velocity, and history of contact. It means frictional stress controls seismic motion. An earthquake can occur only if friction decreases rapidly with slip causing slip weakening. If friction increases with slip, or does not drop rapidly enough, slip motion either stops or occurs gradually.

The tectonic loading stress accumulates until it exceeds the frictional stress; sliding begins; the loading stress drops below that of friction; the fault motion stops; and the process 
repeats. "Sticking" requires that the loading stress be less than the frictional stress. This can occur in the midst of an earthquake because of geometrical and compositional heterogeneity within the fault plane. Both small-scale spatial variations in frictional properties and dynamic effects control the physics of seismic slip on a microscopic level. However, earthquakes are inherently large events with slip displacements as large as $10 \mathrm{~m}$ and particle velocities up to $3 \mathrm{~m} / \mathrm{s}$. The irregular distribution of slip reflects both the complexity of dynamic frictional stress and the heterogeneity of local strength on the fault surface.

\section{SEISMOLOGICAL VARIABLES}

In Earth's crust there are planes that can support only relatively low stresses before rupturing. These weak planes are called fault planes. In a simple model, an earthquake is precipitated when the stress on a fault plane exceeds the static frictional stress, $\sigma_{0}$. Plates on either side of the fault experience a relative displacement, or slip, D over an area $\mathrm{S}$. The displacement may be as great as $10 \mathrm{~m}$, but the linear dimension of a fault in the direction of the displacement is typically tens of kilometers so that the contact area is essentially unchanged as the plates move. As sliding commences, the frictional stress drops to a lower kinetic stress, $\sigma_{\mathrm{f}^{*}}$ The drop is not instantaneous; over the time during which friction drops, the plates slip a distance, Dc, as illustrated in the graph at the bottom. When Dc is small, the dynamic stress drop, $\Delta \sigma_{\mathrm{d}}=\sigma_{0}-\sigma_{\mathrm{f}}$, initially drives the sliding. The sliding stops when the shear stress drops below a final frictional stress, $\sigma_{1}$. A variety of mechanisms can stop sliding, such as geometric and compositional heterogeneity, and dynamically changing velocity- or history-dependent friction, and so $\sigma_{1}$ is not necessarily equal to $\sigma_{\mathrm{f}}$. The difference between initial and final frictional stresses is the static stress drop, $\Delta \sigma_{\mathrm{s}}=\sigma_{0}-\sigma_{1}$.

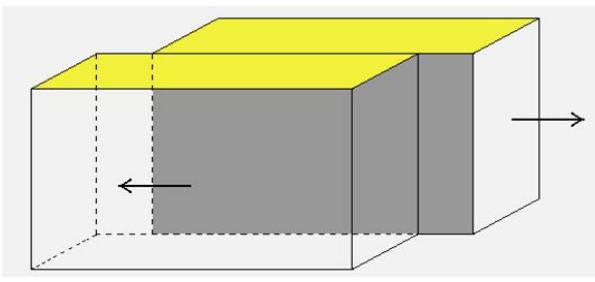

Figure: 1 Fault creation due to the movement of tectonic plates as indicated by arrows.

With seismological methods, one can determine D, S, and the slip velocity, $v_{D}$. Given the rigidity of crustal rocks, $\mu$, and the shear-wave velocity, $\beta$, the dynamic and static stress drops are $\Delta \sigma_{d}=v_{D} \mu / \beta$ and $\Delta \sigma_{s}=\mu_{D} / S 1 / 2$. Both stress drops typically range from 1 to $10 \mathrm{MPa}$, although there are exceptions. Seismological methods measure only transient processes, so they determine stress differences, not the values of the physically important stresses $\sigma_{0}$ and $\sigma_{1}$.

A convenient measure of the overall size of an earthquake is the seismic moment defined by $\mathrm{M}_{0}=\mu \mathrm{SD}$. The seismic moment is measured in energy units, but it does not directly represent the energy released by an earthquake. The magnitude, $\mathrm{M}$, of an earthquake is given in terms of the seismic moment by $\mathrm{M}=\left(\log \mathrm{M}_{0}-9.1\right) / 1.5$.

Slip distributions can be obtained at various times while earthquakes are in progress. Slip is typically heterogeneous in time, just as it is in space. By analyzing slip in both space and time, earthquake scientists can gain insight into rupture dynamics.

\section{RUPTURE DYNAMICS AND ENERGY}

It is very difficult to determine rupture dynamics for irregular fault planes but stress- time histories obtained through rupture patterns are complemented by measurements of total radiated energy, ER, the seismic moment, $\mathrm{M}_{\mathrm{O}}$, which gives the total amount of slip. An earthquake can be viewed as either an instability in frictional contact or the growth of a shear crack. We consider the earth as an elastic solid that can support varieties of wave motions as well as static stress and deformation fields. within this crust are fault planes, zones of weakness along which previous earthquakes have occurred. Due to the large scale tectonic motion of plates, large stresses accumulate within the ground.

It can be imagined the fault as a vertical plane of contact extending from the surface down to about 10 or $15 \mathrm{~km}$. Below this depth, the increased temperature and pressure allow the rocks to move in a ductile manner that prevents the build-up of large stresses. But closer to the surface, the rocks are brittle solids that are being compressed by the enormous weight of the overlying rocks This compressional stress will prevent the two sides of the earth from opening. Since the two sides of the fault are always in contact, there will be a frictional force between them. 


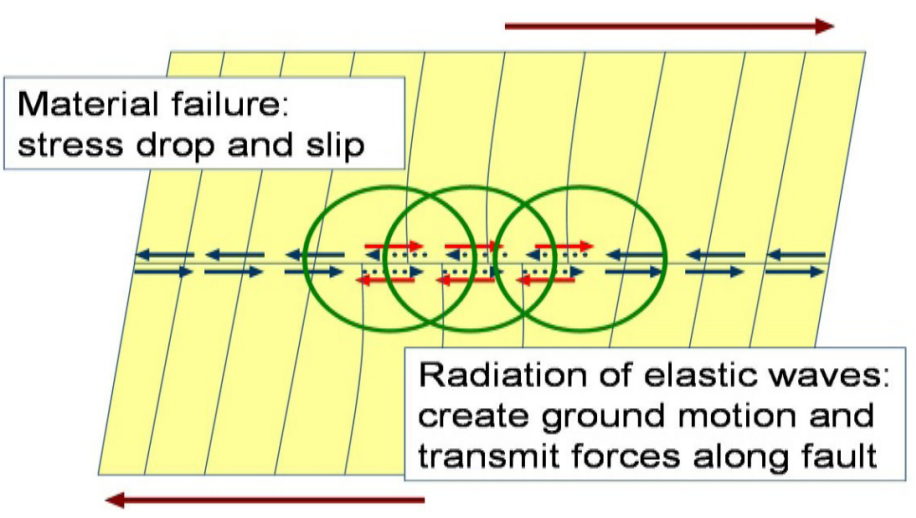

Figure: 3 Material in the fault zone fails and the fault begins to slip. This releases elastic waves, indicated by expanding circles.

For long-term seismic hazard assessment, it would be useful to know how large an area of the fault is close to failure; the size of the earthquake will ultimately be determined by the size of this critical area. It would also be helpful to know just how close the critical area is to failure. In principle, it can be diagnosed by measuring the slope of the magnitudefrequency relationship for the area. (Here "frequency" refers to the occurrence rate of earthquakes.) Although it may be difficult to determine the degree of criticality with seismic data alone, the concept of criticality is important because it suggests the use of other methods to monitor the state of the crust. For example, electromagnetic methods could be used to monitor fluid flow in the crust. When fluid migrates in the crust and weakens some parts of fault zones, the crust could approach a critical state and produce electrical or magnetic noise. If the crust has a low degree of criticality, a small perturbation in stress or weakness is not very likely to cause a large event.

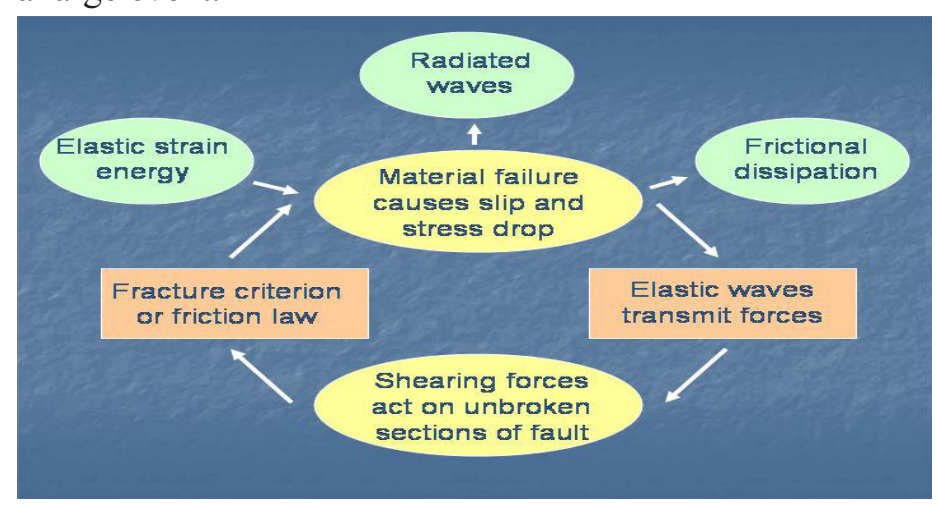

Figure 4: The dynamic rupture process

\section{FAULT LUBRICATION}

Large earthquakes have a substantially greater radiated energy per unit fault area per unit slip than do small earthquakes. It may be due to the fact that large earthquakes typically occur on well-developed, through-going faults and do not use much energy making new surfaces. Small earthquakes, on the other hand, often occur on small cracks and expend a lot of energy creating the new surfaces needed for the crack to grow, leaving less energy to be radiated.

In the presence of fluids, a strictly mechanical effect; elastohydrodynamic lubrication can reduce friction independent of the thermal effect. If the fault zone is thin and rough, and if the material in the fault zone behaves like a viscous fluid, the shearing of the fault fluid produces a viscous stress that is balanced by dynamic pressure. The fluid pressure rises, which both reduces friction and elastically deforms the fault planes. Such elastohydrodynamic lubrication also reduces friction for large events relative to small ones. Thus, when the slip and slip velocity are large and the fault zone is thin, a pressure increase, caused by either thermal or hydrodynamic effects, becomes large enough to smooth out the irregularities on the fault surface by elastic deformation. This smoothing suppresses shortperiod ground motion caused by the fault surfaces rubbing against each other

Lubrication depends on many factors, such as the effective permeability in the fault zone, compressibility of fault rocks, and the viscosity of fault fluid. The thin slip zone is the key concept. If some mechanisms exist to distribute energy over a thick zone (greater than $10 \mathrm{~cm}$ ) during a seismic event, it may be possible to maintain high friction on a fault plane. If the viscosity of the frictional melt is high, melting may actually increase the fault friction. However, in view of the large slip and slip velocity associated with seismic faulting, we believe significant lubrication is likely to occur if the slip zone is thin.

\section{CONCLUSIONS}

The earthquake is a complex process to be explained by a single model of physics. But there are few common parameters that help to design a model and understand the physics of earthquake. The fundamental problem is to understand the microscopic processes using macroscopically observed parameters. One microscopic feature is of particular interest to us: the thin fault zone. During large seismic events, thermal and mechanical processes may result in low friction in such zones. This low friction, combined with estimated 
static and dynamic stress drops, suggests that mature seismic fault systems operate at relatively low stresses, on the order of tens of MPa. On the other hand, the crust supports large surface loads, such as mountains, that require its strength to be at least $100 \mathrm{MPa}$. This means that the stress in the crust is spatially extremely heterogeneous, and the system organizes itself into a somewhat precarious state with low stress on major faults.

During an earthquake, a small stress perturbation can trigger a seismic event. The growth of the earthquake is controlled by heterogeneous friction on the fault, which in turn may be affected by nonlinear processes involved in lubricated dynamic slip. Elastohydrodynamic lubrication in a thin fault zone may change the roughness of the fault plane and thereby change the frequency spectrum of ground motion. Understanding these processes is key to a better understanding of seismicity, rupture dynamics, and ground motion characteristics that lead to an effective seismic risk lessening measures.

\section{REFERENCES}

1. Strahler and Strahler, Elements of physical geography; p-300

2. C. Tsuboi , Bull. Earthquake Res. Inst., U. Tokyo 10, 411 (1932). Google Scholar,

3. E. Rabinowicz, Friction and Wear of Materials, Wiley, New York (1995). Google Scholar,

4. D. J. Wald, T. H. Heaton, Bull. Seismol. Soc. Am. 84, 668 (1994)

5. Andrews, D.J., Dynamic Plane-Strain Shear Rupture with a Slip-Weakening Friction Law Calculated by a Boundary Integral Method, 1985.

6. Beroza, G. C., and P. Spudich, Linearized Inversion for Fault Rupture, J. Geophys. Res., 93, 6275-6296, 1988.

7. Dunham, E. M., P. Favreau, and J. M. Carlson, A Supershear Transition Mechanism for Cracks, Science, 299, 1557-1559, 2003.

8. H. Kanamori and E.E. Brodsky,Rep.Prog. Phys.67(2004) 